\title{
Modifications of Coronay Artery Calcifications (CAC) and Correlations with C Reactive Protein (CRP) Levels in Renal Recipients after the First Year of Transplantation
}

\author{
Paolo De Paolis ${ }^{1}$, Roberto Colonnelli ${ }^{1}$, Antonio Favarò ${ }^{1}$, Massimo Iappelli ${ }^{2}$, Concetta Carriero ${ }^{2}$, \\ Daniela Mantella ${ }^{1}$, Giorgio Coen ${ }^{3}$, Salvatore Di Giulio ${ }^{1}$ \\ ${ }^{1}$ Nephrology Transplant Unit, Polo Ospedaliero Interaziendale Trapianti, A.O. S.Camillo-Forlanini e INMI L. Spallanzani, Rome, Italy \\ ${ }^{2}$ Surgical Transplant Unit, Polo Ospedaliero Interaziendale Trapianti, A.O. S.Camillo-Forlanini e INMI L. Spallanzani, Rome, Italy \\ ${ }^{3}$ Ospedale Israelitico, Rome, Italy \\ Email: pdepaolis3@alice.it
}

Received October 28, 2012; revised February 27, 2013; accepted March 13, 2013

\begin{abstract}
The purpose of the present study was to determine the association between presence and progression of Coronary Artery Calcifications (CAC) quantified with Agatston Score (AS) and inflammatory index as CRP and other parameters in unselected renal transplant recipients. Forty-five patients were underwent a baseline Multislice CT (MSCT) at the time of renal transplant and a repeat evaluation 12 - 16 months later. After second MSCT recipients were divided in three groups: Gr1 (26 patients) with absence of CAC at basal and second MSCT, Gr2 (11 patients) with reduction of CAC after one year and Gr3 (8 patients) with increased values of CAC after one year. Mean $+/-$ Standard deviation of basal and after one year values of AS and CRP were respectively: Gr1: $2+/-3 ; 2+/-5$ and $0.4+/-0.3 ; 0.55+/-0.67$; Gr2: $317+/-288 ; 212+/-242$ and $0.9+/-1.1 ; 0.55+/-0.6$; Gr3: $854+/-1168 ; 1032+/-1153$ and $0.8+/-0.8 ; 1.1+/-$ 0.96 . We found capacity of renal transplantation to protect against development of new calcium deposits in recipients without $\mathrm{CAC}$ at time of transplantation. While we confirmed association in $\mathrm{Gr} 2$ between reduction of CAC with reduction of CRP levels and in Gr3 between increased levels of CRP with increasing of CAC. Conclusion: In this preliminary study, renal transplantation appears to slow down or increasing CAC, in strict association with modifications of CRP levels. Long term studies are needed to confirm our preliminary data and to determine the effects of CAC on cardiovascular morbidity and mortality in renal transplant recipients.
\end{abstract}

Keywords: Coronary Artery Calcifications; Renal Transplant; Inflammation; Agatston Score

\section{Introduction}

Coronary Artery Calcification (CAC) are commonly attributed to the coexistence in uremia of several pathogenetic factors such as derangements of divalent ions metabolism, circulating levels of inhibitory proteins of calcification and inflammation [1-5]. Renal transplantation improves disorders of mineral metabolism and removes many uraemic toxins therefore may improve vascular calcifications, However transplantation is also pro-atherogenic with induction of hyperglycemia/glucose intolerance, hyperlipidemia and hypertension by the various immunosuppressive agents [6]. Prevalence of CAC have assessed in renal transplant recipients [7-11], that are related to age and time of dialysis [12] and other several studies have showed that renal transplantation appears to slow down or arrest the process in most patients. However CAC has the strong capacity of prediction for cardiovascular events in renal transplant patients [13]. Some authors have individuated in hyperparathyroidism and inflammation as factors related to the progression of CAC in these patients [14]. We conducted a study in un-selected asymptomatic renal transplant recipients to determine the association of presence and progression of CAC quantified with Agatston Score (AS) with inflammation index as $\mathrm{C}$ Reactive Protein (CRP) and other determinants in the first year after transplantation.

\section{Material and Methods}

Forty-five unselected and asymptomatic patients with end-stage renal disease in hemodialysis treatment undergoing renal transplantation in our Transplant Center POIT between May 2007 and November 2008, were studied to evaluate the association among coronary calcification scores at the time of transplantation and after one year, and other determinants as CRP after one year of observation. Patients treated for hyperparathyroidism 
with vitamin $\mathrm{D}$ at time of renal transplantation were not excluded. Exclusion criteria were ethanol or drug abuse, clinical cardiovascular disease, chronic inflammatory disease, human immunodeficiency virus infection and use of steroid, antiepilectic drugs, non-steroidal antinflammatory drugs, estrogens or anticoagulants. The patients underwent the baseline Multisclice CT (MSCT) within 1 month after the transplant and they were asked to undergo a second MSCT, 10 - 14 months after the renal transplant. Blood samples for biochemical evaluation were drawn under fasting condition, after one month and after one year after transplantation during clinical follow up. The following assays were made: serum creatinine, calcium, phosphorus, alkaline phosphatase, intact parathyroid hormone $(\mathrm{PTH})$, serum albumin, 25(OH) Vitamine D3 and 1.25(OH $)_{2}$ Vitamin D3, Ostase, Osteocalcin and CRP levels. Total calcium was corrected for serum albumin using equation: total calcium $=\mathrm{Ca}+0.8$ (4.0-albumin). All recipients received a similar immunosuppressive therapy with basiliximab induction, tacrolimus plus mycophenolate mofetil plus steroids. Our immunosuppressive therapy foresees through level of tacrolimus to reach $6-8 \mathrm{ng} / \mathrm{ml}$, steroid dose tapered to $20 \mathrm{mg} / \mathrm{die}$ at third month to reach at six month, stable dose of $5 \mathrm{mg} /$ die. MMF reduced in presence of leucocites reduction. In our therapeutic strategy we don't effect prophilaxis for CMV and we effect immunosuppressive therapy reduction for BKV replication (for urinary and serum values, respectively $>10^{5}$ and $>10^{3}$ copies $/ \mathrm{ml}$ ). After one year of observation, the recipients were divided retrospectively in three groups: Group 1: 26 recipients with absence of calcification at basal time and after 1 year; Group 2: 11 recipients with presence of calcification at basal time and reduction after one year; Group 3: 8 recipients with presence of calcification at basal time and increased after one year (hereafter three groups refered respectively as Gr1, Gr2 and Gr3).

\section{Statistical Analysis}

Data are expressed as mean values $+/-$ standard deviation (SD) or proportion where required while continuos variables are compared with the student $t$ test. A p values $<0.05$ are considered significant.

\section{Results}

The study was carried out 45 recipients undergoing renal transplantation and was followed for one year after transplantation. Patients ( 31 males/14 females) had mean age of $48+/-14$ and median 50 years and hemodialysis vintage of $99+/-104$ and median 70 months. All patients reached end of follow up at 1 year post-transplantation with functional graft. We observed 3 recipients with delayed graft function with need to temporary hemodialysis treatment (mean time +/- SD: $3.6+/-2.2$ weeks and mean number of treatment +/- SD: $11+/-9$ treatment): no acute rejection. After first MSCT we found 26 recipients without coronary calcification (AS: 2 $+/-3.4$; median 0) while 19 (42.2\%) showed basal coronary artery calcification quantified with AS of $516+/-$ 795; median 170. After year of observation, we retrospectively divided patients in three groups in according with modification of AS to second MSCT. Second MSCT showed 26 patients with absence of coronary calcification (Gr1) (AS: $1.5+/-3.3$ and median 0) while other patients with basal coronary calcification showed two different modifications. Of these recipients, 11 recipients with basal AS of $317+/-288$, median 175 (Gr2), showed reduction of AS to $213+/-242$, median 154 ( $\mathrm{p}=$ $0.05)$ while the third group with 8 recipients $(\mathbf{G r 3})$ with basal AS $759+/-1129$ and median 120, showed increased AS values to reach $925+/-1129$ and median 692 $(p<0.02)$. Recipients that at basal time don't present coronary calcification, during the year of study, doesn't present new calcifications. Basal and after 1 year of observation clinical, biochemical data, calcification scores and statistical analysis, of forty-five recipients, separated groups are showed in Table 1. In Gr1 (26 recipients) we found a significant differences only for $25(\mathrm{OH})$ and $1.25(\mathrm{OH})_{2}$ Vitamin D3 (respectively $\mathrm{p}<0.001$ and 0.004 ) while other parameters didn't show a statistical differences. In Gr2 (11 recipients) we found only statistical differences in serum osteocalcin $(\mathrm{p}<0.04)$ and AS $(\mathrm{p}<$ $0.05)$ while other parameters didn't show statistical differences. In Gr3 (8 recipients) we found statistical differences in serum phosphorus $(\mathrm{p}<0.04)$, CRP levels $(\mathrm{p}<$ $0.02)$ and AS $(p<0.02)$. Other parameters didn't show statistical differences. For inflammation, our results didn't show the CRP related to presence (basal time) and modification for CAC (after 1 year) in Gr1. Our study showed, after 1 year, CRP significant differences versus AS of G2 and G3 (respectively p $<0.01$ and $p<0.01$ ) (Figure 1) and we found strict association between age of recipients and CRP values in Gr2 and Gr3 vs Gr1 (respectively p < 0.001 and $\mathrm{p}<0.0001$ ) while we didn't association between Gr2 and Gr3 (pNS) as sign of association of elevated CRP levels and age in Gr2 and Gr3.

Multivariate analysis, for the reduced number of patients of single group, don't reach statistical differences.

\section{Discussion}

The aetiology of vascular calcification in CKD -5 patients is multifactorial. Older age and longer time of dialysis are prominent risk factors in a number of studies assessing vascular calcification by various techniques [7,15-18] and other studies have demonstrated that dyslipidaemia, systolic hypertension and disorders of 

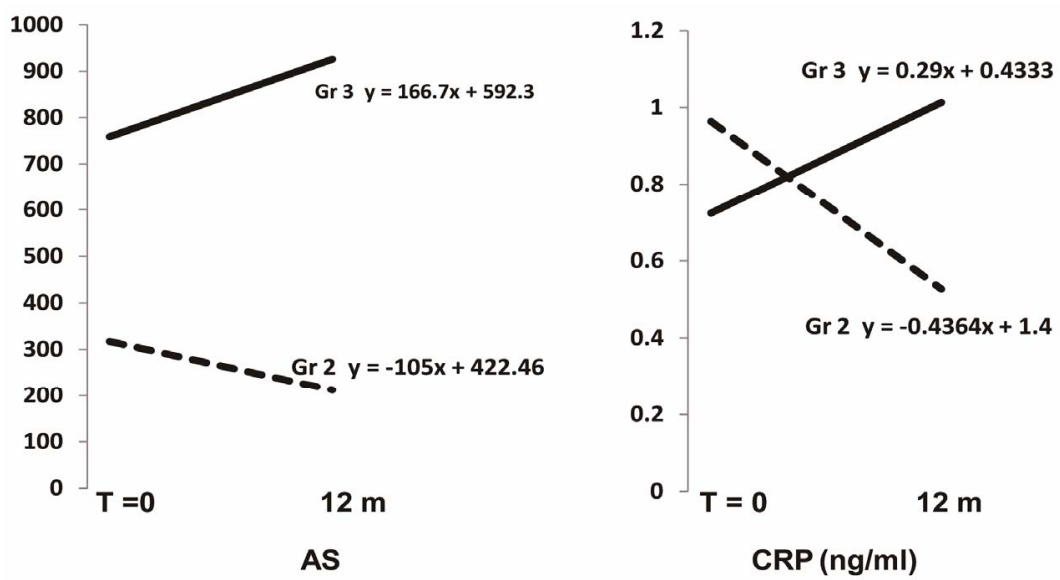

Figure 1. Modifications of C Reactive Protein and Agatston Score values after 12 months of renal transplantation in Gr2 and Gr3.

Table 1. Baseline characteristics and results of the 45 patients included in the study.

\begin{tabular}{|c|c|c|c|c|c|c|c|c|c|}
\hline & Gr1: 26 pts & & & Gr2: 11 pts & & & Gr3: 8 pts & & \\
\hline & $1 \mathrm{~m}$ & $1 y$ & $p$ & $1 \mathrm{~m}$ & $1 y$ & $p$ & $1 \mathrm{~m}$ & $1 y$ & $p$ \\
\hline Don Age (y) & $38+/-15$ & & & $54+/-11$ & & & $50+/-16$ & & \\
\hline Rec Age (y) & $41+/-13$ & & & $56+/-10$ & & & $59+/-13$ & & \\
\hline Months HD & $69+/-37$ & & & $136+/-103$ & & & $142+/-201$ & & \\
\hline $\mathrm{T}$ isch (h) & $8.4+/-1.8$ & & & $8.2+/-2$ & & & $8.5+/-4.1$ & & \\
\hline S. Creat $(\mathrm{mg} / \mathrm{dl})$ & $1.6+/-0.5$ & $1.4+/-0.3$ & 0.001 & $2.2+/-2.1$ & $1.9+/-0.9$ & NS & $2.4+/-1.3$ & $1.6+/-0.6$ & 0.002 \\
\hline Ca corr (mg/dl) & $9.6+/-0.4$ & $9.6+/-0.3$ & NS & $9.7+/-1.1$ & $9.6+/-0.7$ & NS & $9.7+/-0.9$ & $9.5+/-1.1$ & NS \\
\hline $\mathrm{P}(\mathrm{mg} / \mathrm{dl})$ & $2.5+/-0.6$ & $2.9+/-0.5$ & 0.001 & $2.6+/-1.7$ & $3.1+/-0.6$ & NS & $2.5+/-0.6$ & $3.5+/-1$ & 0.02 \\
\hline $\mathrm{FA}(\mathrm{u} / \mathrm{i})$ & $206+/-63$ & $224+/-86$ & NS & $324+/-188$ & $296+/-139$ & NS & $221+/-54$ & $282+/-183$ & NS \\
\hline PTH i (pg/ml) & $86+/-52$ & $97+/-104$ & NS & $124+/-70$ & $143+/-111$ & NS & $86+/-90$ & $76+/-74$ & NS \\
\hline Osteoc (ng/ml) & $14+/-11$ & $15+/-9$ & NS & $21+/-18$ & $30+/-18$ & 0.02 & $13+/-19$ & $18+/-18$ & NS \\
\hline Ostase $($ microg $/ \mathrm{L})$ & $21+/-13$ & $20+/-9.9$ & NS & $25+/-13$ & $28+/-13$ & NS & $27+/-18$ & $33+/-20$ & NS \\
\hline Vit D $25 \mathrm{OH}(\mathrm{nmol} / \mathrm{L})$ & $43+/-23$ & $59+/-34$ & 0.02 & $50+/-21$ & $81+/-56$ & 0.006 & $44+/-19$ & $55+/-31$ & NS \\
\hline Vit d 1-25OH (nmol/L) & $92+/-35$ & $122+/-40$ & 0.001 & $68+/-39$ & $142+/-49$ & 0.0001 & $89+/-24$ & $123+/-43$ & 0.03 \\
\hline $\mathrm{CRP}(\mathrm{mg} / \mathrm{dl})$ & $0.4+/-0.3$ & $0.5+/-0.7$ & NS & $0.96+/-1.1$ & $0.55+/-0.67$ & 0.06 & $0.8+/-0.8$ & $1.1+/-0.9$ & 0.002 \\
\hline AS & $2+/-3$ & $2.4+/-5$ & NS & $317+/-288$ & $212+/-243$ & 0.002 & $854+/-1168$ & $1038+/-1152$ & 0.01 \\
\hline
\end{tabular}

mineral metabolism including elevated phosphorus and/ or calcium phosphorus product and calcium load in the form of phosphate binders are significant risk factors for vascular calcification [7,16-17]. Aim to our study is to continue the observation started with previous study [19] after 1 year of functional graft and to evaluate the parameters that may be involved in destiny of CAC and to verify the effects of presence of CAC on CRP as sign of inflammatory index. Our results confirm the relationship between CAC detected with MSCT as sign of general- ized arterial calcification and inflammatory index as CRP. Cause of this detection is sure related to elevated time of dialysis prior renal transplantation and the elevated percentage of phosphate binders based on calcium used for therapy. Our study, with the observation of same recipients for the first year of transplantation with functional graft, has confirmed literature data on capacity of renal transplantation to protect against development of new calcium deposits in recipients without CAC at time of transplantation. We agree with literature data on associa- 
tion between inflammatory index as CRP and CAC. In fact in $\mathrm{Gr} 2$ and $\mathrm{Gr} 3$ with presence of CAC at basal time, we found strict association between reduction of CRP and reduction of AS (Gr2) while in Gr3 with CRP increased, we observed increased of AS (Figure 1). Our preliminary study has several potential limitations, the most important of which are: small number of patients, the absence of information of humoral parameters as Fetuin-A and Fibroblast Growth Factor 23, new factors recently found to be involved in vascular calcification (20) and time of study is short to predict effect of CAC on cardiovascular morbidity and mortality in our recipients.

\section{Conclusion}

The preliminary results of our study after 1 year of transplantation confirm the strict correlation between CAC quantified with AS and inflammatory index as CRP. Long term studies are needed to investigate the effect of humoral parameters as Fetuin-A and FGF23 and the effect of CAC on cardiovascular morbidity and mortality in transplant recipients.

\section{REFERENCES}

[1] S. Jono, M. D. McKee, C. E. Murry, et al., "Phosphate Regulation of Vascular Smooth Muscle Cell Calcification," Circulation Research, Vol. 87, 2000, pp. e10-e17. doi:10.1161/01.RES.87.7.e10

[2] H. Yang, G. Curinga, C. M. Giachelli, et al., "Elevated Extracellular Calcium Levels Induce Smooth Muscle Cell Matrix Mineralization in Vitro," Kidney International, Vol. 66, 2004, pp. 2293-2299. doi:10.1111/j.1523-1755.2004.66015.x

[3] K. Lomashvili, P. Garg, W. C. O’Neil, et al., "Chemical and Hormonal Determinants of Vascular Calcification in Vitro," Kidney International, Vol. 69, No. 8, 2006, pp. 1464-1470.

[4] M. Ketteler, P. Borgantz, R. Westenfeld, et al., "Association of Low Fetuin-A (AHSG) Concentrations in Serum with Cardiovascular Mortality in Patients on Dialysis: A Cross-Sectional Study," Lancet, Vol. 361, No. 9360, 2003, pp. 827-833. doi:10.1016/S0140-6736(03)12710-9

[5] P. Stenvinkel, K. Wang, A. R. Qureshi, et al., "Low Fetuin-A Levels Are Associated with Cardiovascular Death: Impact of Variations in the Gene Encoding Fetuin," Kidney International, Vol. 67, No. 6, 2005, pp. 2283-2392.

[6] B. L. Kasisle, "Cardiovascular Disease after Renal Transplantation," Seminars in Nephrology, Vol. 20, 2000, pp. 176-187.

[7] W. G. Goodman, J. Goldin, B. D. Kuizon, et al., "Coronary-Artery Calcifictaqion in Young Adults with EndStage Renal Disease Who Are Undergoing Dialysis," New England Journal of Medicine, Vol. 342, 2000, pp. 1478-1483. doi:10.1056/NEJM200005183422003

[8] M. B. Ishitani, D. S. Milliner, D. Y. Kim, et al., "Early
Subclinical Coronary Artery Calcification in Young Adults Who Were Pediatric Kidney Transplant Recipients," American Journal of Transplantation, Vol. 5, No. 7, 2005, pp. 1689-1693.

doi:10.1111/j.1600-6143.2005.00914.x

[9] P. A. McCullogh, K. R. Sandberg, F. Dumler, et al., "Determinants of Coronary Vascular Calcification in Patients with Chronic Kidney Disease and End-Stage Renal Disease: A Systenmatic Review," Journal of Nephrology, Vol. 17, No. 2, 2004, pp. 205-215.

[10] P. T. Nguyen, E. Coche, E. Goffin, et al., "Prevalence and Determinants of Coronary and Aortic Calcifications Assessed by Chest CT in Renal Transplant Recipients," American Journal of Nephrology, Vol. 27, No. 4, 2007, pp. 329-335. doi:10.1159/000102978

[11] K. Nitta, T. Akiba, K. Suzuki, et al., "Assessment of Coronary Artery Calcification in Hemodialysis Patients Using Multi-Detector Spiral CT Scan," Hypertension Research, Vol. 27, No. 8, 2004, pp. 527-533. doi:10.1291/hypres.27.527

[12] S. E. Rosas, K. Mensah, R. B. Weinstein, et al., "Coronary Artery Calcification in Renal Transplant Recipients," American Journal of Transplantation, Vol. 5, No. 8, 2005, pp. 1942-1945.

[13] P. T. Nguyen, S. Henrard, E. Coche, et al., "Coronary Artery Calcification: A Strong Predictor of Cardiovascular Events in Renal Transplant Recipients," Nephrology Dialysis Transplantation, Vol. 25, No. 11, 2010, pp. 3773-3778.

[14] S. Mazzaferro, M. Pasquali, F. Taggi, et al., "Progression of Coronary Artery Calcification in Renal Transplantation and the Role of Secondary Hyperparathyroidism and Inflammation," Clinical Journal of the American Society of Nephrology, Vol. 4, No. 3, 2009, pp. 685-690. doi: $10.2215 /$ CJN.03930808

[15] J. Braum, M. Oldendorf, W. Moshage, et al., "Electron Beam Computed Tomography in the Evaluation of Cardiac Calcification in Chronic Dialysis Patients," American Journal of Kidney Diseases, Vol. 27, No. 3, 1996, pp. 394-401. doi:10.1016/S0272-6386(96)90363-7

[16] G. M. London, A. P. Guerin, S. J. Marchais, et al., "Arterial Media Calcification in End-Stage Renal Disease: Impact on All-Cause and Cardiovascular Mortality," $\mathrm{Ne}$ phrology Dialysis Transplantation, Vol. 18, No. 9, 2003, pp. 1731-1740. doi:10.1093/ndt/gfg414

[17] G. M. Chertow, S. K. Burke and P. Raggi, "Sevelamer Attenuates the Progression of Coronary and Aortic Calcification in Hemodialysis Patients," Kidney International, Vol. 62, No. 1, 2002, pp. 245-252. doi:10.1046/j.1523-1755.2002.00434.x

[18] S. M. Moe, K. D. O’Neill, N. Fineberg, et al., "Assessment of Vascular Calcifictaions in ESRD Patients Using Spiral CT," Nephrology Dialysis Transplantation, Vol. 18, No. 6, 2003, pp. 1152-1158. doi:10.1093/ndt/gfg093

[19] G. Coen, P. De Paolis, P. Ballanti, et al., "Peripheral Artery Calcifications Evaluated by Histology Correlate to Those Detected by CT: Relationships with Fetuin-A and FGF-23," Journal of Nephrology, Vol. 24, No. 3, 2011, pp. 313-321. 
[20] C. Marechal, G. Schlieper, P. Nguyen, et al., "Serum Fetuin-A Levels Are Associated with Vascular Calcifications and Predict Cardiovascular Events in Renal Trans- plant Recipients," Clinical Journal of the American Society of Nephrology, Vol. 6, No. 5, 2011, pp. 974-985. 